\title{
Rapid dynamics of the microtubule binding of ensconsin in vivo
}

\author{
J. Chloë Bulinski ${ }^{1}$, David J. Odde ${ }^{2}$, Bonnie J. Howell ${ }^{3}$, Ted D. Salmon ${ }^{3}$ and Clare M. Waterman-Storer 3,4 \\ ${ }^{1}$ Departments of Biological Sciences, Anatomy \& Cell Biology \& Pathology, Columbia University, College of Physicians \& Surgeons, 630 W. 168 th \\ St, Rm BB1213, New York, NY 10032-3702, USA \\ 2Department of Biomedical Engineering, University of Minnesota, Minneapolis, MN 55455, USA \\ ${ }^{3}$ Department of Biology, University of North Carolina, Chapel Hill, NC 27599, USA \\ ${ }^{4}$ Cell Biology and Institute for Childhood \& Neglected Diseases, Scripps Research Institute, La Jolla, CA 92037, USA \\ ¥Author for correspondence (e-mail: jcb4@columbia.edu)
}

Accepted 15 July 2001

Journal of Cell Science 114, 3885-3897 (2001) (C) The Company of Biologists Ltd

\section{SUMMARY}

Microtubule-associated proteins (MAPs) are proteins that reversibly bind to and regulate microtubule dynamics and functions in vivo. We examined the dynamics of binding of a MAP called ensconsin (E-MAP-115) to microtubules in vivo. We used $5 \times$ GFP-EMTB, a construct in which the microtubule-binding domain of ensconsin (EMTB) is fused to five copies of green fluorescent protein (GFP), as a reporter molecule amenable to the use of fluorescent speckle microscopy. Fluorescent speckle microscopy (FSM) sequences and kymograph analyses showed rapid dynamics of speckles comprised of $5 \times$ GFP-EMTB in untreated cells. By contrast, in detergent-lysed cytoskeletons, speckles were not dynamic. Since detergentlysed cytoskeletons differ from living cells in that they lack both ATP and dynamic microtubules, we used azide treatment to substantially reduce the level of ATP in living cells and we used Taxol to halt microtubule dynamics. Both treatments slowed the dynamics of $5 \times$ GFP-EMTB speckles observed by FSM. We also used fluorescence recovery after photobleaching (FRAP) to quantify the half-time of binding and dissociation of the $5 \times$ GFP-EMTB chimera and to compare this half-time to that of the full-length MAP molecule. In untreated cells, the $t_{1} / 2$ of either $5 \times$ GFP-EMTB or full-length GFP-ensconsin was similarly rapid ( 4 seconds), while in ATP-reduced and Taxol-treated cells, $t_{1} / 2$ was increased to 210 seconds and 40 seconds, respectively. In detergent-extracted cells no recovery was seen. Consistent with the rapid dynamics of $5 \times$ GFP-EMTB measured with fluorescent speckle microscopy and FRAP, we estimated that the affinity of the MAP for microtubules is $\sim 40 \mu \mathrm{M}$ in untreated living cells, compared with $\sim 1 \mu \mathrm{M}$ in vitro. However, $K_{D}$,app was not significantly changed in the presence of azide and was increased to $110 \mu \mathrm{M}$ in the presence of Taxol. To test whether changes in the phosphorylation state of cellular proteins might be responsible for altering the dynamics of ensconsin binding, we used FSM to monitor staurosporine-treated cells. Staurosporine treatment substantially halted dynamics of $5 \times$ GFP-EMTB speckles along MTs. Our results show that ensconsin is highly dynamic in its association with microtubules, and its microtubule association can be altered by in vivo phosphorylation events.

Key words: Fluorescent speckle microscopy, Photobleaching, Taxol, Microtubule-associated proteins, Phosphorylation, Epithelial cells

\section{INTRODUCTION}

Cells use microtubules (MTs) for intracellular transport, mitotic division, cell motility and cellular morphogenesis. Participation in such a variety of cellular activities involves morphologically diverse MT arrays, including symmetric radial arrays, mitotic spindles or polarized cytoplasmic arrays. Markedly different MT polymerization dynamics are also required for MT-based functions during interphase, compared with mitosis (Saxton et al., 1984; Hamaguchi et al., 1985), and during proliferation, compared with differentiation (Mandelkow and Mandelkow, 1995). Disparate cell types and activities may acquire the requisite morphologies and dynamics of their MT arrays due to the action of non-tubulin components such as MT-associated proteins (MAPs) (Mandelkow and Mandelkow, 1995; McNally, 1999). MAPs, proteins that bind along the length of MTs, but show neither high affinity binding nor enzymatic activity for unpolymerized tubulin, include MAPs of the tau, MAP1 and MAP2 families, which are abundantly expressed in nervous tissue, and appear to modulate MT properties there (Mandelkow and Mandelkow, 1995).

That MAPs contribute to the disparities in dynamics and functions of MTs in cells other than neurons is supported by studies of vertebrate non-neuronal cells and tissues (Cassimeris, 1999; Andersen, 2000), in which the most abundant MAPs, MAP4 (Bulinski and Borisy, 1979; Parysek et al., 1984), e-MAP (Suprenant et al., 1993; Lepley et al., 1999), and ensconsin (E-MAP-115) (Bulinski and Borisy, 1979; Masson and Kreis, 1993), each appear to be functionally distinct. MAP4 is a $210 \mathrm{kDa}$ MAP that has been shown to stabilize MTs and modulate their level in vivo (Olsen et al., 1995; Nguyen et al., 1999). By contrast, e-MAP, a $77 \mathrm{kDa}$ MAP first isolated from echinoderm eggs (Suprenant et al., 
1993), has been shown to promote MT dynamic instability by binding to MTs and decreasing their propensity to re-enter the rescue phase during catastrophic depolymerization (Hamill et al., 1998). Ensconsin, an $84 \mathrm{kDa}$ protein expressed most abundantly in epithelial tissue (Fabré-Jonca et al., 1998) does not appear to modulate the dynamics of MTs in proliferating cells under physiological conditions (Faire et al., 1999). However, one property of ensconsin, namely, its tighter in vitro binding to MTs in the presence of the MT-stabilizing drug, Taxol (Bulinski and Bossler, 1994), distinguishes it from other MAPs expressed in the same cell types.

Since different complements of MAPs are expressed at different cell cycle stages and in different cell types, and since MAPs differ in how they modulate the MTs to which they are bound, one must understand how they bind to and dissociate from MTs in vivo, and how their MT binding is regulated. The in vivo association of some MAPs with MTs has been addressed previously. For example, fluorescence recovery after photobleaching (FRAP) studies showed that MAP2, a MAP known to contribute to MT stability in vitro, had a half-time of MT binding of roughly 5 minutes in neuronal cells (Scherson et al., 1984). By contrast, Olmsted et al., performing similar FRAP studies of MAP2 and also of MAP4, whose MT-binding domains are very similar, found half-times of MT binding of 60 and 44 seconds, respectively, for the two MAPs (Olmsted et al., 1989). In addition, the Olmsted group found evidence of cell cycle regulation of both MAPs; in mitosis the half-times measured were 14 and 17 seconds. A comparison of the rates reported for MAP2 MT-binding dynamics by Scherson et al. and Olmsted et al. suggested that cells regulate the affinity or dynamics of this MAP in different cell types or under different cell cycle conditions (Scherson et al., 1984; Olmsted et al., 1989). Technical difficulties precluded quantification of MAP behavior on individual MTs in the two previous studies of MAP:MT dynamics, possibly limiting accuracy of $t_{1} / 2$ data.

Using fluorescent speckle microscopy (FSM) (WatermanStorer et al., 1998) to image green fluorescent protein (GFP) chimeras of another MAP, CLIP-170, Perez et al. showed that CLIP-170 binds exclusively to the ends of growing MTs in vivo (Perez et al., 1999). Further, CLIP-170's MT association was found to be regulated such that the MAP dissociated rapidly after subsequent growth of the MT. These data provided clues about potential functions of CLIP-170, a MAP that apparently does not function as a MT stabilizer.

In the current study, we examine the behavior of the MAP, ensconsin, in vivo. Our interest in ensconsin was sparked by several experimental results (Bulinski and Borisy, 1979; Masson and Kreis, 1993). First, this MAP is cell-type-specific or -selective; it is most prevalent in epithelial-derived cells, such as differentiated cell types (Masson and Kreis, 1993; Fabré-Jonca et al., 1999) or certain carcinoma cell lines (Gruber et al., 2001). Second, ensconsin appears to bind at uniform levels along the entire length of all cellular MTs, associating concomitant with their polymerization (Faire et al., 1999). At levels found on the MTs in undifferentiated interphase cells, associated ensconsin does not affect MT dynamics detectably (Faire et al., 1999). Finally, ensconsin displays more tenacious in vitro binding to MTs in the presence of Taxol (Bulinski and Bossler, 1994). These previous studies suggest a MAP that rapidly associates with all MTs, yet is unknown in its functions or the regulation of its MT binding.
Accordingly, we examined the affinity and dynamics of ensconsin's association with MTs in vivo, using both FSM and FRAP techniques. Our results demonstrate that ensconsin is highly dynamic in its association with MTs in living cells, with a half-life of MT association of approximately 4 seconds. Our data also demonstrate that reduction of cytoplasmic ATP increases this half-life by at least two orders of magnitude, indicating that cells can regulate ensconsin's MT binding dynamics. The MT-stabilizing drug, Taxol, also stabilized ensconsin binding, altering the half-life of binding by an order of magnitude. Our results have implications for possible functions of ensconsin binding and its alteration in response to cellular events.

\section{MATERIALS AND METHODS}

\section{Materials}

Except where noted, all tissue culture solutions and media were purchased from Life Technologies Inc. (Bethesda, MD), and all chemicals were obtained from Sigma Chemicals (St Louis, MO). Plasticware and other materials were from Fisher Corporation (Springville, NJ).

\section{Cells and treatments}

African green monkey (TC-7) cells expressing 5xGFP-EMTB, a construct in which the MT-binding domain of ensconsin (EMTB) is fused to five copies of green fluorescent protein (GFP), and TC-7 cells expressing GFP-Ensc, a construct encoding full-length ensconsin conjugated to a single GFP, were prepared, characterized, and maintained as described (Bulinski et al., 1999; Faire et al., 1999). In some experiments, TC-7 cells stably expressing $5 \times$ GFP-EMTB and GFP-Ensc were transiently transfected with pDS-red (Faire et al., 1999 ), to provide a fluid phase marker for $K_{\mathrm{D} \text {,app }}$ determinations.

$5 \times$ GFP-EMTB-TC-7 cells were detergent-extracted at $37^{\circ} \mathrm{C}$ in a solution of $0.2 \mathrm{mg} / \mathrm{ml}$ saponin in PEM (80 mM PIPES, pH 6.9, 1 mM EGTA and $1 \mathrm{mM} \mathrm{MgCl} 2$ ) for 3 minutes, then the coverslip was rinsed in PEM and mounted on a slide for microscopy. Other treatments were performed on living cells grown on coverslips: Cells were rinsed once and incubated at $37^{\circ} \mathrm{C}$ in Dulbecco's phosphatebuffered saline containing $5 \mathrm{mM}$ sodium azide and $1 \mathrm{mM}$ deoxyglucose, to substantially reduce cellular level of ATP (Wadsworth and Salmon, 1988). Cells were either imaged after 40120 minutes ATP-reduction or they were allowed to recover from a 60 minute ATP-reduction by rinsing once and incubating in normal culture medium for 40-120 minutes before imaging. In other experiments Taxol ( $1 \mu \mathrm{M}, 120-160$ minutes) or staurosporine ( 25 or $50 \mathrm{nM}, 60-120$ minutes) was added to normal culture medium prior to imaging.

Immunofluorescence was performed as described previously, with rabbit antibodies to detyrosinated tubulin and rhodamine-conjugated secondary antibody. Coverslips were mounted and sealed with VALAP (rather than nail polish, in order to preserve GFP fluorescence for dual wavelength photomicrographs, which were made within 2 days of fixation and staining).

\section{Imaging and calculations}

Imaging of all TC-7-derived cells plated on glass coverslips was performed as described (Waterman-Storer and Salmon, 1997; Faire et al., 1999) on a heated microscope stage $\left(37^{\circ} \mathrm{C}\right)$. For FSM analysis, time-lapse images were collected with an Orca cooled CCD camera (Hamamatsu Photonics, Bridgewater, NJ), and the resultant speckle patterns were used to assess dynamics directly or through kymographs (intensity of line scans over time; created with Metamorph software from Universal Imaging, West Chester, PA). 


\section{Estimation of $K_{\mathrm{D} \text {,app }}$}

We estimated a value for $K_{\mathrm{D} \text {,app }}$ using two methods. In the first, the nocodazole-based approach, $5 \times$ GFP-EMTB-TC-7 cells were microinjected with $\mathrm{X}$-rhodamine tubulin, treated with nocodazole (10 $\mu \mathrm{M}, 12$ hours) to depolymerize the MTs, and images were captured in both GFP and rhodamine channels. We measured the protomer (that is, fluorescence in an area $\sim 1 \times 2 \mu \mathrm{m}$ in size that contained no MTs) after the nocodazole treatment (before release) and after release, that is, following wash-out of the nocodazole on the microscope stage (Faire et al., 1999). MTs with associated $5 \times$ GFP-EMTB reformed completely within 20 minutes, and images of both fluorophores were taken. From these, we estimated the fraction of tubulin in the unpolymerized (protomeric) state. Briefly, we measured the mean intensity of rhodamine in regions of the lamellum clearly devoid of microtubules, and we subtracted background. Measurement of the same region of the cell during nocodazole treatment was used to calculate the ratio of the intensities, to determine the proportion of polymeric tubulin following recovery from drug treatment. The ratio (protomerbefore:protomer $\left.{ }_{\text {after }}\right)$ was $1.67 \quad(n=1$ cell, average of 5 intracellular locations). Since cell morphology did not change appreciably during release from drug treatment, this ratio yields a tubulin protomeric fraction of $1 / 1.67=0.6$; corresponding to a polymeric fraction of 0.4 . In TC-7 cells, we calculated that the total tubulin concentration is $20 \mu \mathrm{M}$, and the tubulin concentration within MTs is $8 \mu \mathrm{M}$, corresponding to a concentration of ensconsin or EMTB binding sites of $8 \mu \mathrm{M}$. To obtain this value, we calculated the amount of non-nuclear protein per cell. Dividing by the approximate cytoplasmic volume yielded a cytoplasmic protein concentration (e.g. $40 \mathrm{mg} / \mathrm{ml})$. Next, the proportion of soluble protein that is tubulin (5\%), determined for TC-7 cells with or without transfected ensconsin constructs (Gundersen et al., 1987; Faire et al., 1999) was used to determine that tubulin $=2 \mathrm{mg} / \mathrm{ml}$ in the cytoplasm. For analogous calculations with similar results, see Larsson et al. (Larsson et al., 1999). As described above for tubulin, we used the ratio of the $5 \times$ GFP-EMTB signal (before:after) drug release to determine that the monomeric and MT-bound fractions of $5 \times$ GFP-EMTB were $1 / 1.86=0.54$, and 0.46 , respectively. The equilibrium dissociation constant, $K_{\mathrm{D}}$, is given by

$$
K_{\mathrm{D}}=\frac{[A][B]}{[A B]},
$$

where $[A]$ is the free concentration of $5 \times$ GFP-EMTB, $[B]$ is the concentration of available binding sites, and $[A B]$ is the concentration of MT-bound $5 \times$ GFP-EMTB. Using FSM images we determined that expression was within the speckle limit (i.e. sufficiently low expression to yield speckles rather than continuous labeling). Thus, $[B]$ is approximately equal to the total binding site concentration $\left([B]_{\text {total }}=[A B]+[B] \sim=[B]\right)$. Additionally, we assumed that virtually all polymerized tubulin molecules are available for binding, because crowding-induced sub-stoichiometric binding would not occur when this low density of $5 \times$ GFP-EMTB is present, and there are no known competitors for the $5 \times$ GFP-EMTB binding site. Therefore, for the cell analyzed by the nocodazole-based approach, the $K_{\mathrm{D} \text {,app }}$ was calculated from

$$
K_{\mathrm{D}}=\frac{0.54}{0.46} * 8=9.4 \mu \mathrm{M} .
$$

We also calculated $K_{\mathrm{D} \text {,app }}$ with a volume marker-based approach, in which cells were transfected with DS-red, a volume marker. Because these cells were not injected with tubulin, the $8 \mu \mathrm{M}$ figure derived above was used in calculating the binding site concentration. As above, background was subtracted and regions devoid of microtubules were identified for analysis. The mean intensity of the volume marker and of $5 \times$ GFP-EMTB in a chosen region $\sim 1 \times 2 \mu \mathrm{m}$ in size (Fig. 4) were measured and their ratio calculated. This ratio, which was constant in all regions of the cell that were devoid of MTs, was used to scale the volume marker to free $5 \times$ GFP-EMTB signal. The scaled volume marker image was subtracted from the total $5 \times$ GFP-EMTB image, leaving an image that was, ideally, proportional to the concentration of MT-bound $5 \times$ GFP-EMTB. A broad region of the lamellum was then identified and the mean intensities of total and bound $5 \times$ GFP-EMTB signal were measured. The ratio, total EMTB:bound EMTB, was used to calculate the fraction bound, the fraction free, and the $K_{\mathrm{D} \text {,app }}$ value, as described above. Two regions were analyzed per cell in each of five cells; results differed by $<10 \%$ within all cells analyzed.

Measurements of $K_{\mathrm{D} \text {,app }}$ in azide- and Taxol-treated cells were made as above, but using MT polymer concentration values of $14 \mu \mathrm{M}$ and $20 \mu \mathrm{M}$, commensurate with previous measurements of TC-7 cells subjected to these MT-stabilizing treatments (Gundersen et al., 1987) (data not shown).

\section{Microscopy and laser photobleaching}

For FRAP analyses, cells expressing $5 \times$ GFP-EMTB were grown on coverslips, placed in a modified Rose chamber, and imaged with a Nikon TE300 inverted microscope equipped with an Orca 1 cooled CCD camera and controlled by MetaMorph imaging software (Faire et al., 1999). Images were obtained using a 100×/1.4 NA Plan Apo Phase 3 objective, a $100 \mathrm{~W}$ mercury arc fluorescent light source (attenuated to $\sim 10 \%$ ), and a Chroma Hy-Q FITC filter set. Photobleaching experiments were performed essentially as described (Maddox et al., 2000). Briefly, a $488 \mathrm{~nm}$ line from an argon laser (Spectra-Physics, Mountain View, CA) was selected through a bandpass filter and shuttered to control exposure. The laser was passed through a beam expander and focused onto the field diaphragm as a spot conjugate to a diameter at half-maximal intensity of $0.8 \mu \mathrm{m}$ at the specimen plane (Maddox et al., 2000). For the experiments shown in Table 1, the laser was intentionally de-focused to illuminate and bleach a spot sufficiently large ( $\geq$ three times larger diameter) to allow separate analysis of FRAP in the edge and middle regions of the bleached spot.

Cells were imaged before and after bleaching. In general, a region on a single MT was bleached, and following recovery, some MTs were bleached and allowed to recover as many as 10 times in the same spot. Images were collected before ( $\geq 30$ seconds) and after ( $\geq 10$ minutes) bleaching, at time intervals of either 1 image per second or by 'streaming', that is, four images collected per second without shutter closure. Exposure times were $100-250 \mathrm{~ms}$ in all cases. Cellular background was subtracted from these data, and the decay of fluorescence (photobleaching) over the same time period in an unbleached portion of the same MT that had been bleached by the laser was fitted with an exponential decay curve $\left[\left(\mathrm{F}_{(\mathrm{t})=}=\mathrm{F}_{0}\left(\mathrm{e}^{-\mathrm{kt}}\right)\right]\right.$, in which $\mathrm{F}_{(\mathrm{t})}$ is the fluorescence at any time, $\mathrm{F}_{0}$ is the initial fluorescence, and $\mathrm{k}$ is the fluorescence decay constant. Decay-corrected MT fluorescence was plotted against time of recovery and fitted to an exponential recovery curve: $F=F_{\text {inf }}-\left(\left(F_{\text {inf }}-F_{b l c h}\right)\left(e^{-k_{O F F}(t)}\right)\right)$ in which $F_{b l c h}$ is the fluorescence at the time of bleaching, and $F_{\text {inf }}$ is the fluorescence at $\mathrm{t}=$ infinity (that is, fluorescence completely recovered). This equation, whose derivation is described completely in Appendix

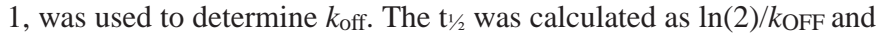
$\%$ recovery was calculated as: $\left[\left(\mathrm{F}_{\text {inf }}-\mathrm{F}_{\mathrm{blch}}\right) /\left(\mathrm{F}_{\text {prebleach }}-\mathrm{F}_{\mathrm{blch}}\right)\right] \times 100$.

\section{RESULTS}

\section{Dynamics of $5 \times$ GFP-EMTB speckles in living cells}

In earlier studies of monkey cells stably expressing a low level of chimeras consisting of several green fluorescent protein (GFP) molecules attached to a single ensconsin MT-binding domain (EMTB), we showed that these chimeras bound along the length of all cellular MTs concomitant with their 


\section{a. untreated}
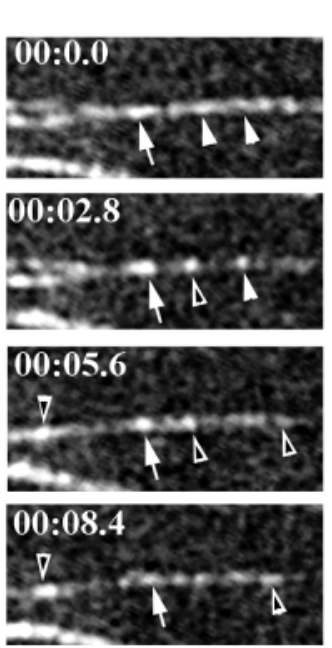

f. untreated

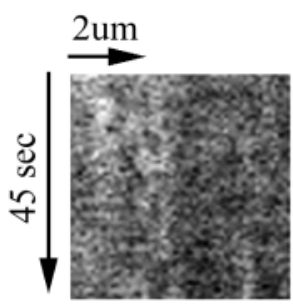

b. detergentextracted
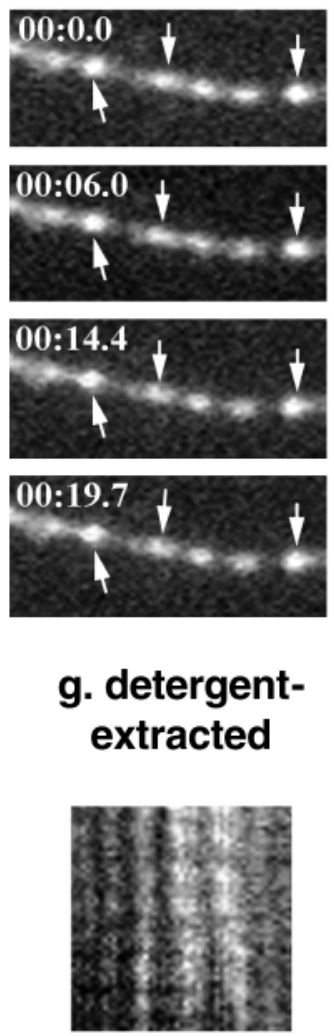

c. ATPreduced
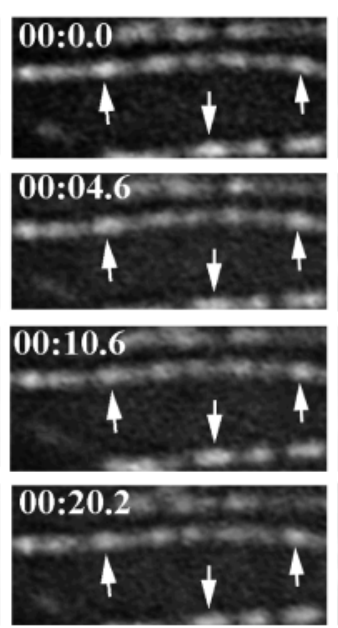

\section{h. ATP- reduced}

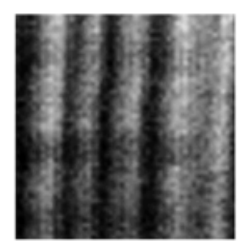

d. ATP- reduced

\& recovered
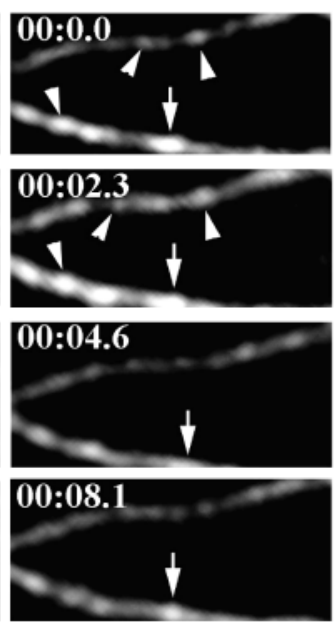
i. ATP- reduced \& recovered

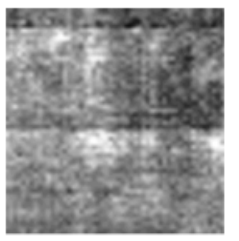

e. Taxoltreated
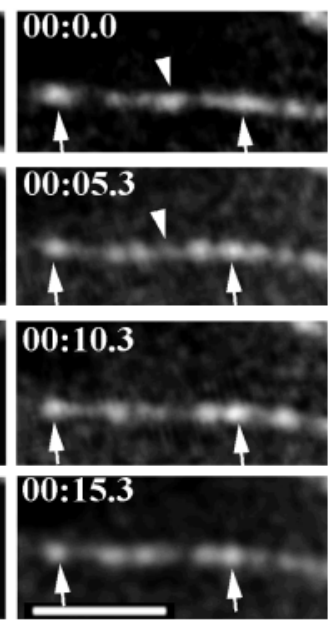

\section{j. Taxol- treated}

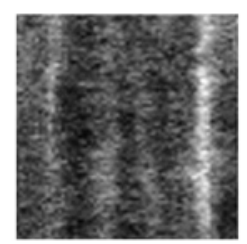

Fig. 1. Dynamics of GFP-ensconsin chimera speckles in vivo. Fluorescent speckle microscopy (FSM) was used to record the dynamics of $5 \times$ GFP-EMTB chimeras in living cells and cytoskeletons. (a-e) Time-lapse micrographs of 5×GFP-EMTB speckles in living cells. Arrows point to speckles that remain from one interval to the next, while arrowheads denote speckles that appear or disappear during the sequence. The bar in the right-hand corner of time-lapse series e shows the magnification of $1 \mu \mathrm{m}$ in all panels. (f-j) Kymographs of a single MT segment from the time-lapse sequences. As shown, a and $\mathrm{f}$ are from untreated, $\mathrm{b}$ and $\mathrm{g}$, are from detergent-extracted, $\mathrm{c}$ and $\mathrm{h}$ are from ATP-reduced, $\mathrm{d}$ and $\mathrm{i}$ are from ATP-reduced and then recovered and e and $\mathrm{j}$ are from Taxol-treated cells. See Materials and Methods for details of the treatments.

polymerization, and did not cause aberrant MT behaviors (Faire et al., 1999). By several criteria, the GFP-EMTB chimeras mimicked the MT-binding behavior of endogenous ensconsin, making them faithful reporters for ensconsin's MTbinding dynamics in living cells (Faire et al., 1999; Bulinski et al., 1999).

We also noted that the fluorescence of chimeras containing four or five GFP molecules per EMTB molecule (4×GFPEMTB and $5 \times$ GFP-EMTB) appeared as discrete fluorescent speckles, rather than continuous label, along MTs (Faire et al., 1999; Bulinski et al., 1999). Accordingly, we made use of fluorescent speckle microscopy (FSM) (Waterman-Storer et al., 1998) to visualize the dynamics of $5 \times$ GFP-EMTB in living cells, as shown in Fig. 1. The time series in Fig. 1a, untreated cells, shows speckles of various apparent sizes and intensities. The fluorescent speckles arise from variation in the number of fluorophores among the $222 \mathrm{~nm}$ resolvable regions along a MT; each fluorophore emits the $510 \mathrm{~nm}$ green light characteristic of GFP. Comparing speckle density along individual MTs with the molar ratio we measured ( $5 \times$ GFP-EMTB:tubulin) suggested that the tiniest speckles represent single molecules of chimera, while the brighter speckles represent stochastically clustered groups of three to five molecules (Bulinski et al.,
1999) (data not shown). Fig. 1a shows that the $5 \times$ GFP-EMTB speckles are highly dynamic. In the example shown, only one speckle present at the start of the time-lapse recording remained in the same position 8 seconds later (Fig. 1a, white arrow). By contrast, speckles at some positions disappeared during the excerpted recording (Fig. 1a, white arrowhead), while some speckles appeared de novo during the recording (Fig. 1a, filled arrowhead). A kymograph made from analysis of a single MT is shown in Fig. 1f. In this visual representation, no obvious pattern of speckles can be observed over time, suggesting that the MAP is highly dynamic in its association with the MT.

In contrast to its behavior in living cells, $5 \times$ GFP-EMTB speckles in detergent-extracted cytoskeletons were not dynamic, indicating a long-lived association of $5 \times$ GFP-EMTB with the MT lattice. Numerous speckles remained in the same relative position throughout the recording (marked by arrows in the time-lapse micrographs, Fig. 1b); these appear as white vertical stripes in the kymograph in Fig. 1g. That extraction halted speckle dynamics was not surprising, since previous experiments had demonstrated that ensconsin remains bound to MTs in detergent-extracted cytoskeletons (Masson and Kreis, 1993; Bulinski and Bossler, 1994). 
Detergent-extracted cytoskeletons differ from living cells in two important features: they are depleted in ATP and soluble proteins relative to living cells, and their MTs do not undergo polymerization/depolymerization dynamics. We tested whether cellular energy and/or MT polymerization dynamics were required for MT-binding dynamics of $5 \times$ GFP-EMTB in living cells. Wadsworth and Salmon demonstrated that treatment of cells with sodium azide and deoxyglucose was sufficient to deplete $70-90 \%$ of cellular ATP within 5-15 minutes, and to halt energy-requiring processes during mitosis (Wadsworth and Salmon, 1988). 5×GFP-EMTB-TC-7 cells treated with sodium azide and deoxyglucose for 40-120 minutes yielded speckle dynamics qualitatively similar to those of extracted cells (compare Fig. 1c,h with Fig. 1b,g). Effects of ATP-reduction were reversible; restoring ATP level by refeeding cells azide-free medium produced substantial speckle dynamics once again (Fig. 1d,i).

To test if speckle dynamics were altered by dampening MT dynamics, we treated cells with Taxol $(10 \mu \mathrm{M})$ (Liao et al., 1995), which markedly slowed speckle dynamics (Fig. 1e,j, note arrows in 1e), although less so than in ATP-reduced or extracted cells. Some speckles in Taxol-treated cells still appeared to be dynamic (note arrowhead in the first two panels of Fig. 1e). Even though their mechanism of action is distinct from that of Taxol, low concentrations of microtubule depolymerizing drugs also dampen cellular MT dynamics, effectively by kinetically stabilizing the MTs (Jordan et al., 1992; Liao et al., 1995). However, we saw no change in speckle dynamics in cells treated with various concentrations of nocodazole or vinblastine (10-200 nM), suggesting that MT dynamics, per se, may not be involved in regulating speckle dynamics. Instead, Taxol may specifically affect ensconsin's binding to the MT lattice via another mechanism.

\section{FRAP measurements of $5 \times$ GFP-EMTB}

FSM analysis showed that speckles of $5 \times$ GFP-EMTB exhibited a short dwell time at each position on the MT (Fig. 1a,f). Rapid speckle dynamics might result if the MAP transiently associated with and dissociated from the MT. Alternatively, speckles might actually move if $5 \times$ GFP-EMTB molecules laterally diffuse along the surface of the MT, perhaps owing to low binding affinity. We used fluorescence recovery after photobleaching (FRAP) measurements to allow us to quantify the $t \frac{1}{2}$ of the MAP chimeras on the MT and to use the pattern of FRAP to distinguish between the dissociation/re-association and lateral diffusion models. As shown in typical images in Fig. 2A, FRAP occurred very rapidly along single $\mathrm{MTs}$ throughout the bleached region in untreated cells. Fig. 2B shows fluorescence quantified along a single MT that has been bleached twice; for each bleach the

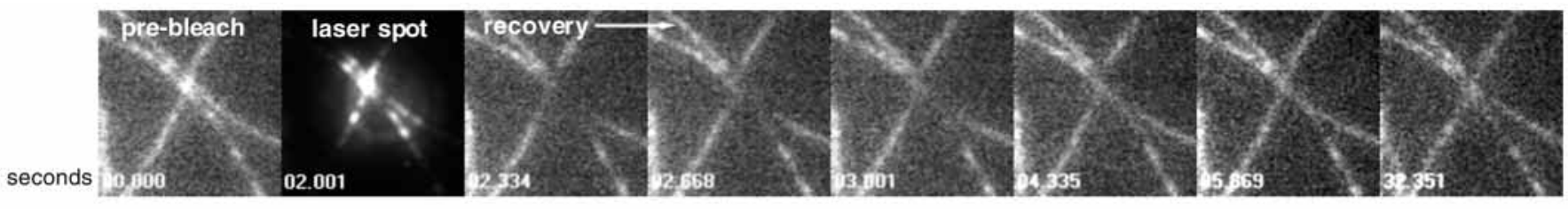

A. Photobleaching and recovery of 5xGFP-Ensc-MTB

\section{B. Corrected fluorescence measurements}

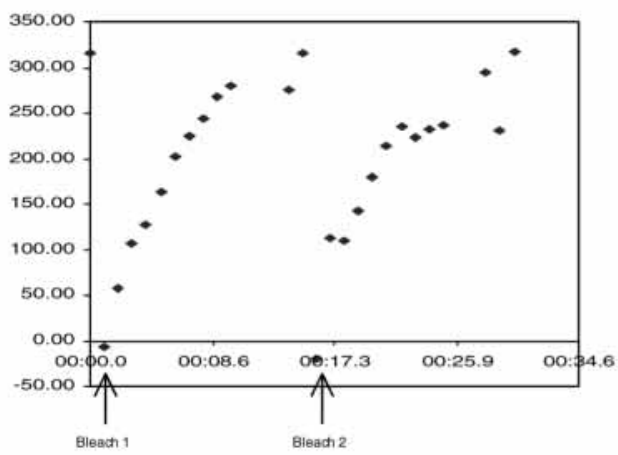

Fig. 2. Photobleaching and recovery of ensconsin chimera in untreated cells. (A) Time-lapse micrographs of the bleached area before (pre-bleach) at the time of bleaching (laser spot) and at intervals during the recovery. Elapsed time, in seconds, is shown in each micrograph. (B) Corrected fluorescence (total pixel intensity along a segment of a bleached MT) was plotted against time (in seconds) before and after each of two bleach events. The time of each bleach is marked with an arrow. Fluorescence shown has been corrected, using an unbleached segment of an adjacent MT, for decay caused by photobleaching during time-lapse imaging and cellular background has been subtracted. (C) Determination of $\mathrm{k}_{\mathrm{OFF}}$. As described in Materials and Methods, the logarithm of the fraction of fluorescence recovered was plotted against time and the slope, kOFF, was determined by linear regression.

\section{Determination of $\mathbf{k}_{\mathrm{OFF}}$}
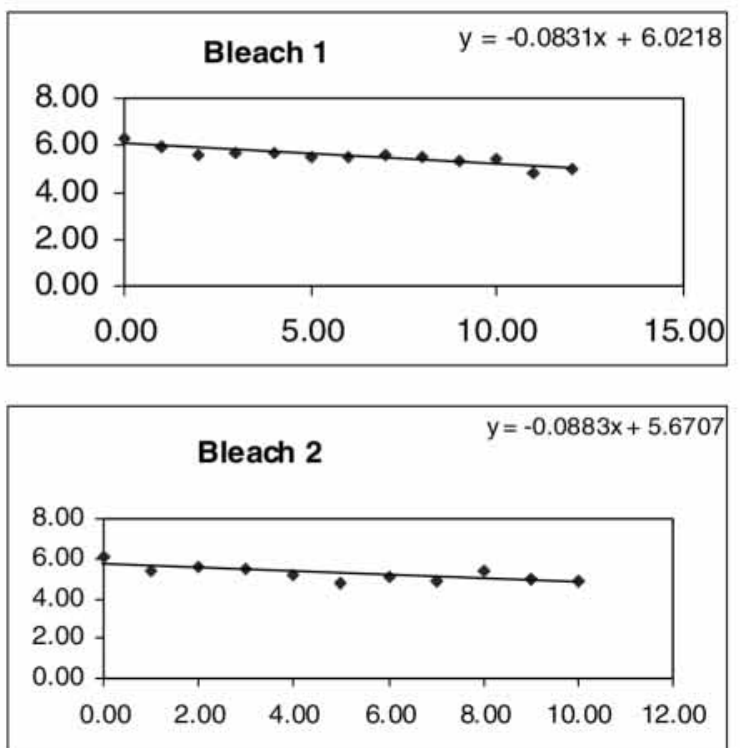
Table 1. FRAP dynamics do not vary with position along the MT

\begin{tabular}{|c|c|c|c|c|c|}
\hline Ensconsin chimera & MT area measured & kOFF $\mathrm{s}^{-1 *}$ & $\mathrm{t}_{1} / 2(\mathrm{~s})$ & $\%$ Recovery* & $n$ \\
\hline $5 \times$ GFP-EMTB & Middle of bleached zone & $0.163 \pm 0.122$ & 4.26 & $86.4 \pm 28.8$ & 4 \\
\hline $5 \times$ GFP-EMTB & Edge of bleached zone & $0.189 \pm 0.105$ & 3.67 & $82.6 \pm 26.2$ & 5 \\
\hline
\end{tabular}

*Values represent means \pm s.d. No s.d. is given for $\mathrm{t} 1 / 2$ because it is calculated from measured values of kOFF.

$P=0.8$ for comparisons of $\mathrm{k}_{\mathrm{OFF}}$ in the middle versus the edge of the bleached zone.

fluorescence was corrected for decay due to repeated illumination, using an unbleached segment of MT, and cellular background was subtracted. The graphs in Fig. $2 \mathrm{C}$ show the $k_{\mathrm{OFF}}$ determined from the data in Fig. $2 \mathrm{~B}$, using the equation whose derivation is described in Appendix 1. Figs 2B and 2C show that recovery was similar after each bleaching event.

As shown in Table 1 , the $\mathrm{t}_{1} / 2$ of FRAP, 4 seconds, was indistinguishable for areas in the middle or at the edge of the zone bleached by the laser; recovery was virtually complete in both regions. The fact that we did not observe more rapid recovery at the edge than at the middle of the bleached zone is not consistent with a lateral diffusion mechanism. Instead, these data strongly favor the dissociation/re-association model for speckle dynamics.

Next, we quantified the MT-binding dynamics of $5 \times$ GFPEMTB and full-length ensconsin labeled with a single GFP (GFP-Ensc) (Faire et al., 1999), to determine if MT-binding dynamics of both molecules were quantitatively similar. Our concern was that in vivo dynamic behavior might be altered by the exclusion of $\sim 75 \%$ of the ensconsin sequence and/or the inclusion of five appended GFP moieties in the $5 \times$ GFP-EMTB chimera. As shown in Table 2, both molecules showed equivalent FRAP behavior; each had a $t \frac{1}{2}$ of $\sim 4$ seconds. This result corroborated previous results that suggested that $5 \times$ GFPEMTB faithfully mimics in vivo and in vitro binding of the full-length MAP (Bulinski et al., 1999). Thus, measuring the dynamics of $5 \times$ GFP-EMTB is a valid model for the MTbinding dynamics of full-length ensconsin. The fact that both molecules show identical behavior upon detergent extraction suggests that full-length ensconsin undergoes the same changes in behavior as $5 \times$ GFP-EMTB during various treatments. This result suggests that treatments that change FSM speckle dynamics of $5 \times$ GFP-EMTB would effect similar changes to the dynamics of full-length ensconsin.

Accordingly, we used FRAP to quantify changes in MTbinding dynamics of $5 \times$ GFP-EMTB in cells that were detergent-extracted or subjected to in vivo treatments. As shown in the example in Fig. 3A, after bleaching MTs in detergent-extracted cells we observed negligible FRAP, corroborating the images of static speckles obtained in FSM
(Fig. 1C). Fig. 3B shows the corrected fluorescence of the bleached MT; because there was no recovery $(5.9 \pm 5.8 \%$ recovery, on average), we did not attempt to calculate a $t \frac{1}{2}$ from these data (Table 2). In cells in which ATP was reduced by incubation in azide-containing medium, dynamics were greatly dampened compared with those of untreated cells $\left(\mathrm{t}_{1} / 2=209\right.$ seconds). However, in contrast to extracted cells, substantial FRAP did occur in ATP-reduced cells. Note that ATPreduction was completely reversible (Table 2); FRAP measurements of cells allowed to recover from ATP-reduction were indistinguishable from those of untreated cells.

Results of FRAP from Taxol-treated cells were significantly different from those of untreated cells (Table 2), although the difference was less striking than results from ATP-reduced cells. Taxol-treatment increased the $t_{1} / 2$ of binding tenfold over untreated cells, to 39 seconds. We note that this change in $t_{1} 12$ occurred during a relatively short Taxol treatment ( 2 hours), in which Taxol elicited neither mitotic entry nor cell cycle arrest.

\section{Affinity of $5 \times$ GFP-EMTB for MTs in vivo}

The rapid dynamics of $5 \times$ GFP-EMTB measured via FSM and FRAP suggested that the $5 \times$ GFP-EMTB molecule might bind loosely to MTs in vivo. Further, we hypothesized that an increase in affinity of the MAP is a possible mechanism by which the residence time of the MAP could be increased in cells treated with azide or with Taxol. To test this hypothesis, we used a volume marker-based approach to estimate the dissociation equilibrium constant, $K_{\mathrm{D} \text {,app, }}$ under three different conditions (untreated, ATP-reduced, and Taxol-treated (see Fig. 4 and Materials and Methods for details). In untreated cells, $K_{\mathrm{D} \text {,app }}$ was estimated to be $44 \mu \mathrm{M}$ (Table 3 ). This value, almost 30 fold larger than the in vitro $K_{\mathrm{D} \text {,app }}$ of full-length ensconsin $\left(K_{\mathrm{D}}=1.6 \mu \mathrm{M}\right)$ (Bulinski and Bossler, 1994), is reasonable given the rapid in vivo dynamics shown by FSM and FRAP (Fig. 1A,B; Fig. 2) (Table 1). However, we did not detect a significant change in $K_{\mathrm{D} \text {,app }}$ in azide-treated cells, and in Taxol-treated cells, $K_{\mathrm{D} \text {,app }}$ increased to $110 \mu \mathrm{M}$ (Table 3 ). These results do not support the hypothesis that treatment-induced alteration in the MT residence time of ensconsin chimera can be attributed to changes in ensconsin's affinity for MTs.

Table 2. FRAP dynamics vary with treatment conditions, not chimera used

\begin{tabular}{|c|c|c|c|c|c|c|}
\hline MAP chimera & Treatment condition & $\mathrm{kOFF}^{-1 *}$ & $\mathrm{t}_{1 / 2}(\mathrm{~s})$ & $\%$ Recovery & $P$ value ${ }^{\ddagger}$ & $n$ \\
\hline GFP-Ensc & Untreated & $0.20 \pm 0.09$ & 3.56 & $87.8 \pm 37.9$ & 1.0 & 4 \\
\hline $5 \times$ GFP-EMTB & Untreated & $0.18 \pm 0.11$ & 3.78 & $96.8 \pm 15.6$ & - & 9 \\
\hline $5 \times$ GFP-EMTB & Detergent-extracted & ND & ND & $5.9 \pm 5.8$ & ND & 4 \\
\hline $5 \times$ GFP-EMTB & ATP-reduced & $0.0033 \pm 0.0032$ & 209 & $30.9 \pm 12.4$ & 0.001 & 4 \\
\hline $5 \times$ GFP-EMTB & ATP-reduced \& recovered & $0.19 \pm 0.13$ & 3.69 & $83.0 \pm 24.7$ & 0.9 & 14 \\
\hline $5 \times$ GFP-EMTB & Taxol-treated & $0.018 \pm 0.015$ & 39.3 & $46.9 \pm 25.1$ & 0.0005 & 8 \\
\hline
\end{tabular}

*Values represent mean \pm s.d. No s.d. is given for $\mathrm{t}_{1} / 2$ because it is calculated from measured values of $\mathrm{k}_{\mathrm{OFF}}$.

$\ddagger P$ values were determined by comparing values for k $\mathrm{OFF}$ for each treatment condition to values for untreated $5 \times \mathrm{GFP}$-EMTB (shown in row 2 ).

$\mathrm{ND}$, values for $\mathrm{k}_{\mathrm{OFF}}$ and $\mathrm{t} \frac{1}{2}$ were not determined, since no fluorescence recovery was observed. 

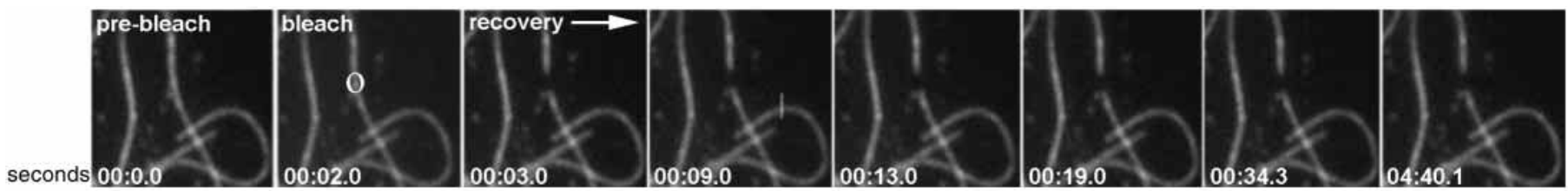

\section{A. Photobleaching and recovery of 5xGFP-Ensc-MTB}

\section{B. Corrected fluorescence measurements}

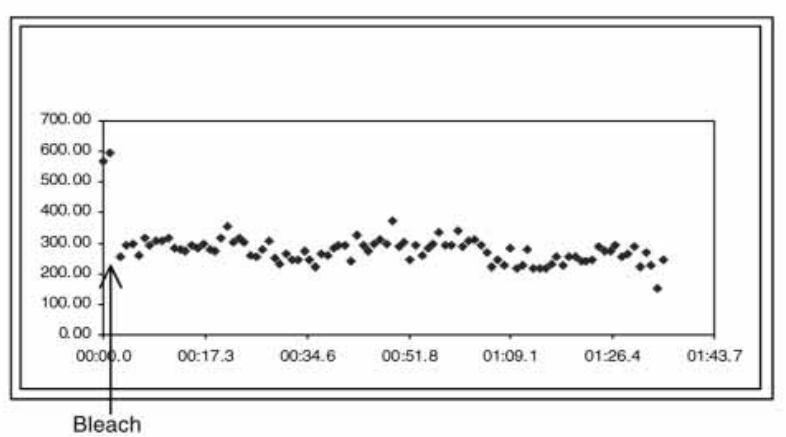

Fig. 3. Photobleaching and recovery of ensconsin chimera in detergent-extracted cells. (A) Time-lapse micrographs of the bleached area are shown before (prebleach), at the time of bleaching (white circle, showing the position of the laser bleach, since no image was captured at the instant of the bleach) and at intervals during the recovery. Elapsed time, in seconds, is shown in each micrograph. (B) Corrected fluorescence of a bleached MT (pixel intensity) was plotted against time (in seconds) at intervals before and after bleaching. The time of the bleach is marked with an arrow; fluorescence shown has been corrected, using an unbleached segment of an adjacent MT, for decay caused by photobleaching during repeated illumination, and cellular background has been subtracted.

\section{Dynamics of $5 \times$ GFP-EMTB does not depend on post-translational detyrosination of MTs}

Alterations in either the MTs or in the MAP chimera, itself, could be responsible for altering MAP:MT binding dynamics in cells that were detergent-extracted, ATP-reduced, or Taxoltreated. Changes in the MT lattice or MAP-binding sites could alter the $t \frac{1}{2}$ of ensconsin binding. One plausible, reversible change in the MTs would be increased tubulin posttranslational modification. All three treatments have been reported to increase the longevity of MTs. Since the detyrosinating enzyme acts on the MT polymer, the longer life of MTs in each treatment condition therefore increases their level of tubulin post-translational detyrosination (Gundersen et al., 1987). If ensconsin chimeras bound more stably to Glu MTs, increasing the detyrosination of all cellular MTs would increase the MT residence time of $5 \times$ GFP-EMTB.

To determine whether an increase in MTs enriched in detyrosinated (Glu) MTs could account for altered binding dynamics of ensconsin chimeras, we subjected $5 \times$ GFP-EMTBTC-7 cells to each treatment employed in FSM and FRAP experiments, and immunostained them with anti-Glu tubulin antibody. We visualized fluorescence of both GFP-EMTB and Glu tubulin; as shown in Fig. 5 we determined that Glu MTs comprised only a subset of MTs under all treatment conditions.

Table 3. Estimation of $K_{D \text {,app }}$ for $5 \times$ GFP-EMTB in vivo

\begin{tabular}{|c|c|c|c|c|c|c|}
\hline Condition & $\begin{array}{l}\mathrm{K}_{\mathrm{D}, \text { app }} \\
(\mu \mathrm{M})^{*}\end{array}$ & $\begin{array}{c}\mathrm{K}_{\mathrm{D}, \text { app }}(\mu \mathrm{M}), \\
\text { upper } 95 \% \text { confidence limit }\end{array}$ & $\begin{array}{c}\mathrm{K}_{\mathrm{D}, \text { app }}(\mu \mathrm{M}), \\
\text { lower } 95 \% \text { confidence limit }\end{array}$ & $\begin{array}{l}\Delta \mathrm{G}^{\mathrm{o}} \text { app } \\
\left(\mathrm{k}_{\mathrm{B}} \mathrm{T}\right)\end{array}$ & $n^{\S}$ & $P^{\mathrm{II}}$ \\
\hline Untreated & 11 & 44 & 2.7 & -11.4 & 5 & - \\
\hline ATP-reduced & 34 & 170 & 6.6 & -10.9 & 5 & 0.05 \\
\hline Taxol-treated & 46 & 110 & 19 & -10.9 & 5 & 0.005 \\
\hline
\end{tabular}

* $\mathrm{K}_{\mathrm{D} \text {,app }}$ shown was estimated using the volume-marker approach described in Materials and Methods. Although only a single, untreated cell was analyzed using the nocodazole-based approach, results were similar to those presented here; the latter method yielded a K $\mathrm{D}_{\mathrm{D}}$ app of $9.4 \mu \mathrm{M}$ (see Materials and Methods for details).

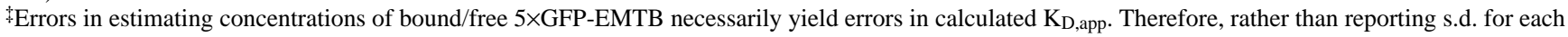
$\mathrm{K}_{\mathrm{D} \text {,app }}$ value, we report here the most extreme values for $\mathrm{K}_{\mathrm{D} \text {,app }}$ that we calculated from $5 \times \mathrm{GFP}$-EMTB values, bound and free, using high and low values. Thus, the $95 \%$ confidence limits were estimated by multiplying the s.d. of the $\Delta \mathrm{G}^{\mathrm{o}}$ app estimates by 2 , adding this energy to the mean $\Delta \mathrm{G}^{\mathrm{o}}$ app to obtain the upper $95 \%$ confidence limit in $\Delta \mathrm{G}^{\mathrm{o}}$ app, and subtracting this energy to get the lower $95 \%$ confidence limit in $\Delta \mathrm{G}_{\text {app. }}^{\mathrm{o}} \mathrm{Note}$ that $\mathrm{K}_{\mathrm{D} \text {,app }}(\mathrm{M})$ and $\Delta \mathrm{G}^{\mathrm{o}}$ app $\left(\mathrm{k}_{\mathrm{B}} \mathrm{T}\right)$ are related through $\Delta \mathrm{G}^{\mathrm{o}}{ }_{\mathrm{app}}=\mathrm{ln} \mathrm{K}_{\mathrm{D}}$, allowing us to calculate the upper and lower $95 \%$ confidence level of $\mathrm{K}_{\mathrm{D} \text {,app. }}$

$\S$ All measurements were made in five separate cells; two areas per cell were measured, and the area of the centrosome was avoided.

IProbability that treatment values were the same as control, determined by two-tailed $t$-test assuming equal variance. Note that the increase in $\mathrm{K}_{\mathrm{D} \text {,app }}$ with Taxol is statistically significant, while the change with azide is borderline $(P=0.05)$. 
Note that some increase in Glu MTs occurred, especially in Taxol-treated cells (compare Fig. 5a and 5g). However, even after Taxol treatment, numerous MTs in the array were not enriched in Glu tubulin. In addition, we noted that Glu MTs were no more prevalent in extracted cells (Fig. 5c), on which $5 \times$ GFP-EMTB showed the greatest $\mathrm{t}_{1} / 2$, than in ATP-reduced (Fig. 5e) or Taxol-treated cells (Fig. 5g); in fact the converse appeared to be true. Since FSM and FRAP dynamics were altered on all MTs examined, but only some MTs in treated cells were enriched in Glu tubulin, altered binding of $5 \times$ GFP-EMTB to Glu MTs, compared with their tyrosinated counterparts, cannot explain the change in MT binding dynamics of the MAP. Since we are aware of no rapid, reversible change in the MTs that alters all MTs concomitant with each of the three treatments, $5 \times$ GFP-EMTB's altered MT-binding dynamics most likely result from a change in the MAP, itself, rather than in the MT.

Modification of MAP phosphorylation state might be expected in cells that have been extracted, ATP-reduced or Taxol-treated. However, other ATPrequiring proteins, instead or in addition, could be active in changing ensconsin behavior. Other proteins have been shown to be changed in their phosphorylation state by Taxol (Wolfson et al., 1997; Shtil et al., 1999; Blagosklonny et al., 1999; Yujiri et al., 1999; Stone and Chambers, 2000). To test whether phosphorylation might indeed play a role in ensconsin behavior, we treated cells with staurosporine, a general inhibitor of protein kinases. We chose treatment conditions sufficient to inhibit most cellular protein kinases (Sauve et al., 1999) but insufficient to cause major cellular damage such as induction of apoptosis (Desagher et al., 1999). We analyzed speckle dynamics of $5 \times$ GFP-EMTB by FSM in the presence of staurosporine. Fig. 6A shows time-lapse images of a sample MT from a staurosporine-treated cell, and Fig. 6D shows the corresponding kymograph. We noted that $5 \times$ GFP-EMTB speckles were markedly static on the MTs in staurosporine-treated cell, in contrast to the dynamic speckles seen on MTs in untreated cells (Fig. 6B). Speckles on MTs in staurosporine-treated cells looked similar to those in ATPreduced cells (compare Fig. 6A and C), suggesting that FRAP data from staurosporine-treated cells would also give results similar to ATP-depleted cells. The ability of staurosporine treatment to dampen the dynamics of $5 \times$ GFP-EMTB speckles suggests that kinase activity is required for the rapid dynamics of ensconsin's association with MTs in vivo. Thus, alteration

\section{5xGFP-EMTB DS-red (Volume)}
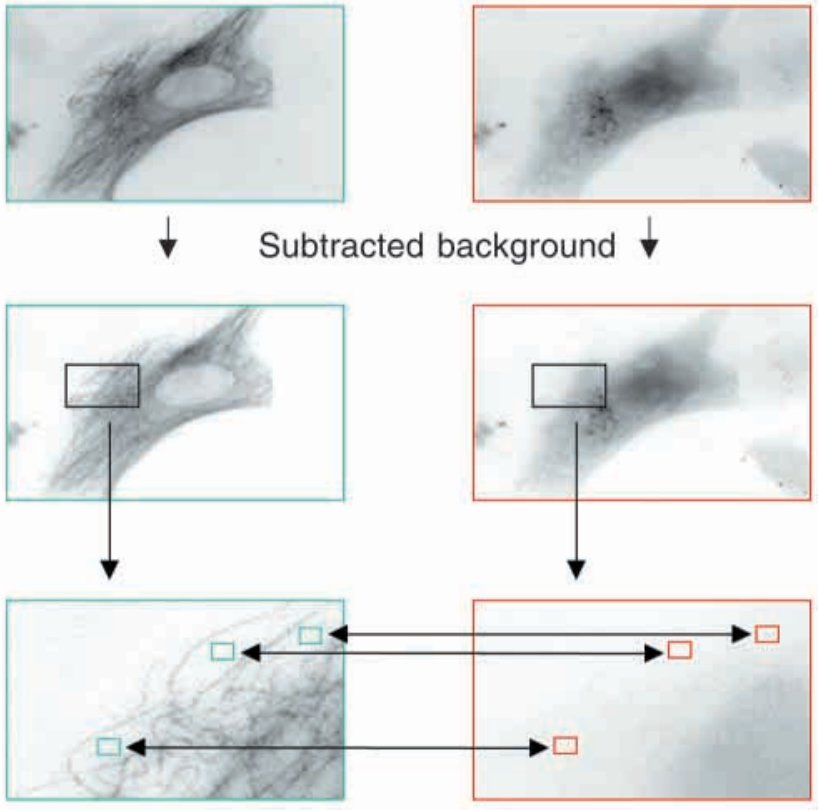

Paired regions that were devoid of microtubules were used to scale DS-red image to obtain image of unbound GFP

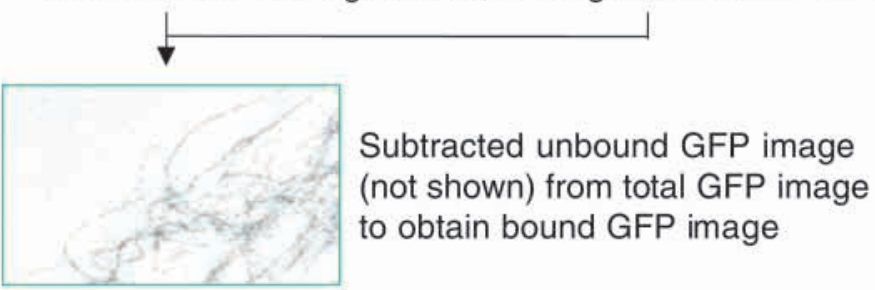

Fig. 4. Measurement of bound and unbound $5 \times$ GFP-EMTB in living cells. In order to estimate Using soluble DS-red expressed in the same cells was used as a volume marker. As shown in the figure, images of GFP and DS-red were captured from the same cell. Next, in each image, (sound fluorescence was subtracted from an area that contained MTs and regions free of MTs (shown in box). To quantify unbound $5 \times$ GFP-EMTB, paired regions that were devoid

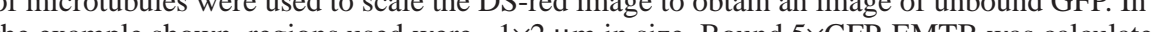
as (Total GFP) - (Bound GFP). Bound and unbound $5 \times$ GFP-EMTB were used to calculate the apparent dissociation constant, $K_{\mathrm{D} \text {,app} \text {. }}$

in the phosphorylation state of the MAP chimera most readily explains the alteration in MAP:MT dynamics we observed during treatment with detergent, azide, or Taxol.

\section{DISCUSSION}

In this paper, we documented extremely rapid dynamics of ensconsin's MT-binding ( $\mathrm{t}_{1 / 2} \sim 4$ seconds). Our results show that ensconsin's dynamics are even more rapid than had been inferred from previous data demonstrating that the MAP associates and dissociates concomitant with the growth and shrinkage of individual MTs (Faire et al., 1999). Our results also demonstrate that ensconsin's in vivo MT-binding dynamics are at least tenfold more rapid than the figures of 60 seconds and 44 seconds that were reported for MAP4 and 


\section{5xGFP-EMTB Glu tubulin}
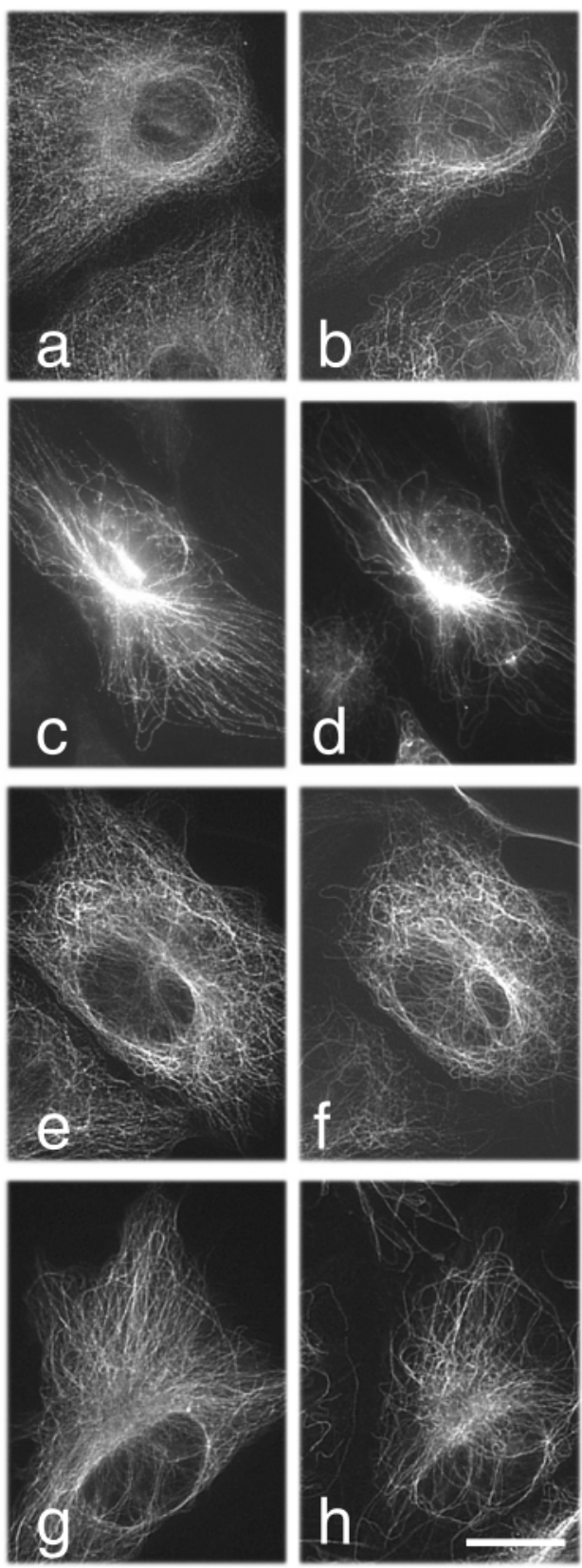

Fig. 5. Dynamics of MAP interaction with MTs does not depend upon their state of post-translational detyrosination. Micrographs of fixed TC-7 cells show fluorescence of MAP chimera visualized with GFP(a,c,e,g) and immunofluorescence of detyrosinated tubulin (antiGlu antibody) visualized with rhodamine secondary antibody(b,d,f,h). Cells were treated as follows: a,b, untreated; c,d, detergent-extracted; e,f, ATP-reduced (azide-treated); and g,h, Taxoltreated. Note that, although there are more detyrosinated MTs in treated cells than in untreated ones, the detyrosinated MTs constitute only a subset of the total array under all conditions. Bar, $10 \mu \mathrm{m}$.

MAP2, respectively (Olmsted et al., 1989) in a different line of epithelial cells, and >100-fold more rapid than the figure of $\sim 5$ minutes that was reported for MAP2 in differentiated cells (Scherson et al., 1984). Direct measurements of in vivo dynamics of tau protein have not been reported, but Mercken et al. used data showing that the axonal transport rate of tau differed from the transport rate of other MT components to suggest that tau may be slightly more dynamic in its in vivo MT association than MAP2 (Mercken et al., 1995). Bearing in mind differences in cell types as well as MAPs investigated in other studies, we conclude that the in vivo MT association/dissociation of ensconsin appears to be more dynamic than that of any other MAP known to bind along the length of MTs.

Olmsted et al. suggested that changes in the cell cycle or differentiation state might influence MAP:MT binding dynamics (Olmsted et al., 1989). Certainly, changes in phosphorylation state and MT dynamics that occur during cell cycle progression and during differentiative events would appear to be sufficient to change ensconsin's MT-binding dynamics. In untreated cells, in which ensconsin exhibits a dynamic association with MTs, it does not appear to influence MT dynamics (Faire et al., 1999). However, our results suggest that ensconsin is functionally altered and may contribute to MT stability under conditions in which it exhibits a long-lived, rather than a transient, association with MTs. Fabré-Jonca et al. demonstrated increased expression of ensconsin (E-MAP115) during epithelial differentiation; they hypothesized that the MAP contributes to the MT stabilization that accompanies differentiation (Fabré-Jonca et al., 1998; Fabré-Jonca et al., 1999). This is a reasonable hypothesis, providing that conditions extant during cell differentiation are sufficient to slow the dynamics of the MAP's MT association, and that its resulting, prolonged association then contributes to MT stability. Under in vitro conditions in which ensconsin was tightly bound to Taxol MTs, the MAP appeared to contribute to stabilization of these MTs against depolymerization by calcium (Bulinski and Bossler, 1994). Whether ensconsin stabilizes MTs in vivo when bound in its less dynamic state is a subject for future investigation.

Our results suggest that changes in the MTs themselves are unlikely to explain changes in ensconsin:MT binding. Alterations in binding dynamics more likely result from changes in ensconsin itself. For example, it is unlikely that changes in MT dynamics are sufficient to alter the residence time of ensconsin on MTs, since we saw no change in speckle dynamics in cells treated with concentrations of nocodazole that were previously reported to dampen MT dynamics by inhibiting assembly/disassembly at plus ends (Jordan et al., 1992; Liao et al., 1995). In addition, ensconsin's MT-binding dynamics are unlikely to be responsive to the quantity of MT polymer, since treatment with Taxol increases the level of MT polymer in cells, while detergent extraction does not, yet both treatments dampen the speckle dynamics of $5 \times$ GFP-EMTB. Similarly, altered ensconsin binding is unlikely to be attributable to changes in the MT lattice, since the lattice is unlikely to be changed in the same way, and to different extents, by each of the four treatments that alter ensconsin binding.

A reasonable hypothesis is that ensconsin:MT dynamics are dictated, at least partially, by the MAP's in vivo affinity for MTs. The affinity of the ensconsin chimera, $5 \times$ GFP-EMTB, for MTs was estimated to be roughly $40 \mu \mathrm{M}$ (Table 3). This value is not unexpected because it suggests that ensconsin binds less tightly in vivo than in vitro, where its affinity was 


\section{FSM Recordings}

Fig. 6. In vivo MT-binding dynamics of GFPensconsin chimeras are reduced by treatment with staurosporine. Fluorescent speckle microscopy (FSM) was used to record the dynamics of $5 \times \mathrm{GFP}-$ EMTB chimeras in living cells. (A,B,C) Time-lapse micrographs of $5 \times$ GFP-EMTB speckles in living cells, treated with (A) staurosporine ( $25 \mathrm{nM}, 2$ hours), a general inhibitor of protein kinases; (B) no treatment; and (C) sodium azide and deoxyglucose ( $5 \mathrm{mM}$ and $1 \mathrm{mM}, 30$ minutes) to substantially reduce cellular ATP. Arrows indicate speckles that remain from one panel to the next; open, white arrowheads denote speckles that disappear during the time-lapse sequence; and black-filled arrowheads denote speckles that appear during the time-lapse sequence. Elapsed time of each exposure is shown in seconds under each panel. (D-F) Kymographs of a MT segment from each of the time-lapse sequences; the scale markers on the left indicate the distance and time over which the kymographs were made. Kymographs and micrographs are the same magnification. Note that treatment with staurosporine substantially reduced dynamics of $5 \times$ GFP-EMTB speckles (A,D), in comparison with untreated cells $(\mathrm{B}, \mathrm{E})$.

\section{B. no treatment}

A. staurosporinetreated

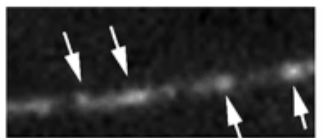

00.00 .0

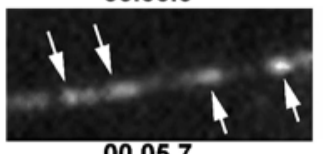

00.05 .7

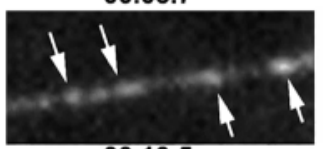

00.13 .5

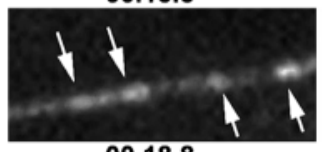

00.18 .8

D.

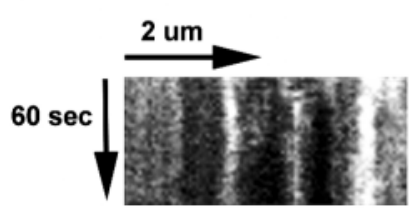

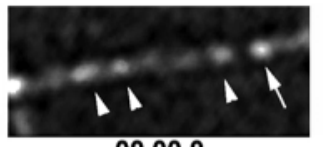

00.00 .0

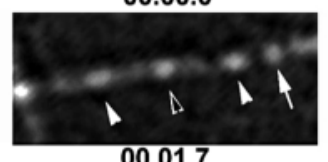

00.01 .7

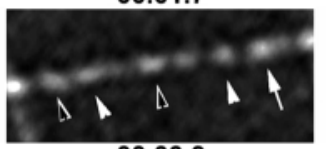

00.03 .9

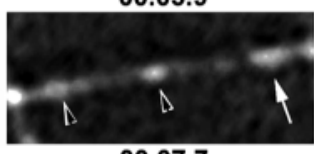

00.07.7

Kymographs

E.

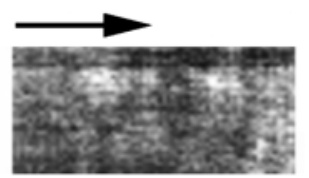

C. ATP-reduced
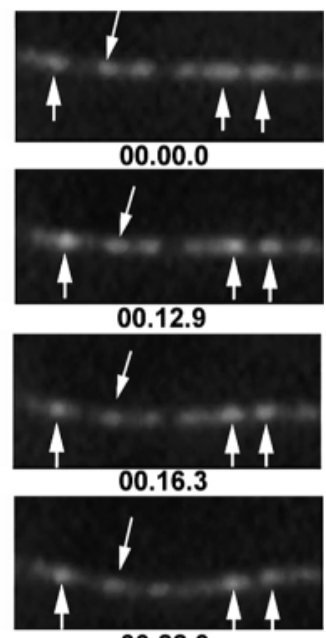

00.22 .3

F.

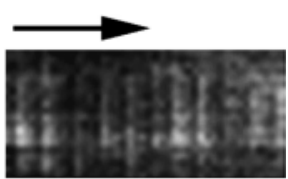

measured to be $1.6 \mu \mathrm{M}$ (Bulinski and Bossler, 1994). In vitro, ensconsin's MT affinity resembled that reported for other MAPs bound along the MT; in vivo affinities of other MAPs could not be compared since only ensconsin's $K_{\mathrm{D} \text {,app }}$ has thus far been estimated in living cells. In vitro affinities of tau protein and MAP4, whose MT-binding domains are highly homologous to one another, are $0.9 \mu \mathrm{M}$ and $1.1 \mu \mathrm{M}$ (Butner and Kirschner, 1991; Chapin and Bulinski, 1994). MAP2, whose MT-binding domain is homologous to those of tau and MAP4, shows an affinity of $5 \mu \mathrm{M}$ for full-length MAP2 (Wallis et al., 1993) and 1.1 $\mu \mathrm{M}$ for its MT-binding domain (Coffey and Purich, 1995). While it is unlikely that ensconsin occupies MT-binding sites that overlap with these other MAPs, because ensconsin and MAP4 were shown not to compete for MTbinding (Bulinski and Borisy, 1980), ensconsin's intrinsic affinity for its own MT-binding sites appears to be similar to the binding of other MAPs to their sites.

We hypothesized that alterations in MT-binding dynamics of ensconsin in cells undergoing Taxol or azide treatments could be explained by changes in the MAP's affinity. However, we were unable to detect a significant change in $K_{\mathrm{D} \text {,app }}$ in azidetreated cells, and in Taxol-treated cells, affinity decreased, rather than increased (Table 3). At first glance, these results appeared difficult to reconcile with FRAP results showing that the Taxol and azide treatments decreased $k_{\mathrm{OFF}}$ by one and two orders of magnitude, respectively (Table 2). However, several mechanisms are consistent with these data. Two feasible models by which $k$ OFF could change without a compensatory decrease in $K_{\mathrm{D} \text {,app }}$ are shown in Fig. 7. In the first, the 'enzyme phosphorylation model', an enzyme promotes both MT association and dissociation of ensconsin, and this catalyst is regulated by a high turnover phosphorylation. The cartoon shows that this enzyme, whose phosphorylation would be inhibited by Taxol and azide, might normally facilitate on/off dynamics of ensconsin by lowering the activation energy for the ensconsin:MT binding reaction. Since the phosphorylated enzyme is required to facilitate both reactions, inhibition of the enzyme's phosphorylation would change binding dynamics, but would not change the relative quantities bound and unbound. Thus, slower dynamics would not decrease the $K_{\mathrm{D} \text {,app. }}$

The second model in Fig. 7, the 'ensconsin phosphorylation model', couples a cycle of ensconsin phosphorylation/ dephosphorylation with the MAP's MT binding/dissociation. As shown in the cartoon, this model envisions that ensconsin binds to the MT in the dephosphorylated state, followed by phosphorylation that enhances its dissociation from the MT. Free ensconsin's phosphate then undergoes hydrolysis prior to rebinding of the MAP to the MT. As shown, this model would predict a change in $K_{\mathrm{D} \text {,app, when }}$ Taxol or azide were applied. However, $K_{\mathrm{D} \text {,app }}$ would not change if the phosphatase that acts on ensconsin was also inhibited by azide or Taxol treatment. In the presence of Taxol or azide, altered association of the phosphatase with ensconsin, or failure to activate the phosphatase by phosphorylation, are equally likely mechanisms by which the ensconsin phosphorylation model would allow a change in $k$ OFF without a compensatory change in $K_{\mathrm{D} \text {,app. }}$ Further studies on ensconsin phosphorylation will be 


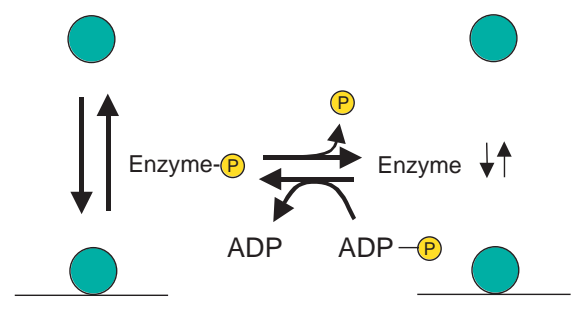

A. Enzyme phosphorylation model

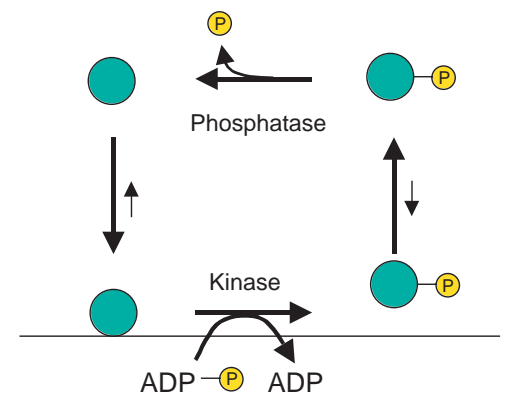

\section{B. Ensconsin phosphorylation model}

Fig. 7. Kinase activity regulates ensconsin's MT-binding dynamics in living cells. Two possible models by which kinase activity could affect the dynamics of ensconsin's association with MTs in vivo. (A) The enzyme phosphorylation model involves a hypothetical enzyme that, when phosphorylated, promotes both ensconsin's association with and dissociation from the MT. Note that this model predicts that ATP depletion or inhibition of protein kinases would bring about a decrease in ensconsin's MT-binding dynamics, without a concomitant increase in MT-binding affinity. (B) The ensconsin phosphorylation model predicts that phosphorylation of ensconsin that occurs while it is bound to the MT enhances its dissociation, while dephosphorylation in the cytoplasm enhances its rebinding to the MT. This model would predict that ATP depletion or inhibition of protein kinases would decrease ensconsin's MT-binding dynamics; an increase in MT-binding affinity of ensconsin would be expected to ensue unless the phosphatase shown was also dependent upon phosphorylation for its activity.

required to define and test these or other mechanisms and to identify components responsible for changing ensconsin's MTbinding dynamics in vivo.

We note that each of the treatment conditions found to change ensconsin:MT dynamics have been correlated with changes in the phosphorylation state of cellular proteins. For example, reduction of cellular ATP level and extraction of cells are both known to change phosphorylation level of many proteins, and Taxol treatment has been reported to alter the activity and phosphorylation state of numerous proteins (Wolfson et al., 1997; Shtil et al., 1999; Blagosklonny et al., 1999; Yujiri et al., 1999; Stone and Chambers, 2000). Since in our current studies we are measuring an early effect of Taxol ( 2 hours) in cells that remain in interphase, we have successfully separated those Taxol effects that are directly due to the drug from those that result secondarily, from Taxolinduced mitotic arrest (Ling et al., 1998). Taken together, results of ATP-reduction, kinase inhibition, extraction, and Taxol-treatment suggest that ensconsin may be dynamically associated with MTs when it is constantly phosphorylated, while its partial dephosphorylation may be sufficient to slow its MT-binding dynamics. Ensconsin phosphorylation may be a switch that the cell uses to regulate the dynamics of the MAP's association with MTs. Moreover, this switch may regulate ensconsin's capacity to stabilize MTs. Our studies may help to uncover a mechanism by which the MT cytoskeleton is reversibly stabilized in vivo.

Although we have not yet identified molecules that interact with the projection domain of ensconsin, it is important to consider that any putative ensconsin-binding proteins would be regulated in their residence time on MTs in concert with the regulation of ensconsin. The recent identification of multifunctional proteins containing domains of interaction for more than one cytoskeletal filament system, such as MACF (Sun et al., 2001) suggests the need to separately regulate the binding to each filament system. Our studies on the regulation of ensconsin may provide a useful paradigm for teasing out independent regulation of binding of these multi-functional proteins to MTs, microfilaments and intermediate filaments.

It is worth noting recent improvements in methodology that facilitated our studies. For example, Olmsted et al. and Scherson et al. were only able to obtain a low level of fluorescent labeling by microinjecting fluorochrome-labeled MAP preparations into cells (Olmsted et al., 1989; Scherson et al., 1984). Low intensity and rapid bleaching of the fluorescence limited their analyses to FRAP studies of specific regions of the cell, or bundles of MTs, respectively. Our use of GFP labeling yielded some improvement and allowed us to analyze single MTs. However, with only a single GFP molecule attached, the fluorescence intensity and bleaching properties of expressed GFP-Ensc made extensive analysis of single MTs difficult. Using the $5 \times$ GFP-EMTB construct, containing five tandemly arrayed GFP's attached to a single molecule of ensconsin's MT-binding domain was a significant improvement that proved to be amenable to FRAP analysis of single MTs, with a high signal-to-noise ratio. Perhaps surprisingly, by several assays, $5 \times$ GFP-EMTB behaved identically to the full-length MAP, confirming its use as a reporter for ensconsin's MT-binding behavior.

In addition, use of the $5 \times$ GFP-EMTB construct as a reporter of ensconsin's MT-binding dynamics allowed us to use FSM for rapidly examining qualitative changes in ensconsin's MTbinding behavior. FSM has been shown to be useful for analysis of actin and tubulin (Waterman-Storer and Salmon, 1998; Waterman-Storer et al., 1998) and of an intriguing MAP, CLIP-170, which associates only with the growing ends of MTs (Perez et al., 1999). The ease of recording FSM observations advanced our knowledge of ensconsin's behavior, demonstrated that its residence time on the MT could be manipulated by experimental conditions, and identified those conditions that should be examined via the more laborious FRAP technique. $5 \times$ GFP-EMTB also allowed us to determine the occupancy of ensconsin binding sites on MTs, and allowed us to estimate, for the first time, the binding affinity of a MAP along MTs in vivo.

Our results also bear on the mechanism by which MTs are stabilized in detergent-extracted cytoskeletons. It was first reported by Bershadsky and Gelfand that addition of ATP was sufficient to depolymerize MTs in detergentextracted cytoskeletons (Bershadsky and Gelfand, 1981). 
Phosphorylation of MAPs was suggested as a potential mechanism for inducing depolymerization of the MTs in these preparations. Our results suggest ensconsin as a candidate MAP that may mediate the stability of extracted cytoskeletons and allow their instability in the presence of ATP. It is notable that detergent-extracted cytoskeletons are a frequent tool for studying MT-based transport or endocytic processes. ATP reduction, extraction of kinases, and cessation of MT dynamics that accompany the preparation of these cytoskeletons are all widely acknowledged to alter the metabolic state of cytoskeletal preparations, relative to living cells. However, it has not been appreciated that ensconsin, and perhaps other molecules with similar behavior, may cause cytoskeletal preparations to be markedly different in molecular composition from the in vivo MT cytoskeleton that is being modeled. This is especially worrisome, since increased amounts of other MAPs, tau and MAP4, on in vivo MTs have been shown to be sufficient to inhibit various MT-based transport events (Bulinski et al., 1997; Ebneth et al., 1998). Likewise, decreasing the $k_{\text {off }}$ of ensconsin binding may be sufficient to alter transport events, rendering detergent-extracted cytoskeletons or even some in vivo models inappropriate for study of many transport events. Cells may even co-opt this mechanism for regulating MT-based transport in vivo.

\section{APPENDIX}

\section{Relation of the FRAP recovery rate constant to the dissociation rate constant, $k_{\text {off }}$}

Assuming that molecule $A$ can bind reversibly to a spatiallyfixed site $B$ to form a complex $A B$, then the reaction equilibrium is given by

$$
A+B \underset{k_{\text {off }}}{\stackrel{k_{\text {on }}}{\rightleftarrows}} A B
$$

The forward reaction rate (units: $\mathrm{M} \mathrm{s}^{-1}$ ) is

$$
r_{\mathrm{on}}=k_{\mathrm{on}}[A][B]
$$

where $k_{\text {on }}$ is the bimolecular association rate constant (units: $\mathrm{M}^{-1} \mathrm{~s}^{-1}$ ) and the reverse reaction rate (units: $\mathrm{M} \mathrm{s}^{-1}$ ) is

$$
r_{\mathrm{off}}=k_{\mathrm{off}}[A B]
$$

where $k_{\text {off }}$ is the unimolecular dissociation rate constant (units: $\mathrm{s}^{-1}$ ) and all the bracketed quantities are the molar concentrations of the indicated species. At equilibrium these two rates are equal $\left(r_{\text {on }}=r_{\text {off }}\right)$ and the corresponding association equilibrium constant, $K$, is defined by

$$
K=\frac{k_{\mathrm{on}}}{k_{\mathrm{off}}}=\frac{[A B]_{e}}{[A]_{e}[B]_{e}}=\frac{1}{K_{\mathrm{D}}}
$$

where the subscript ' $e$ ' indicates the concentration at equilibrium. Given the reaction in Eqn A1, the rate of formation of $A B$ at any arbitrary time is given by

$$
\frac{d[A B]}{d t}=k_{\mathrm{on}}[A][B]-k_{\mathrm{off}}[A B] .
$$

In the FRAP experiments, we use a brief laser pulse to photobleach the fluorescent species $A$ when it is bound to $B$, so that at time $\mathrm{t}=0,[\mathrm{AB}]$ will be 0 . We assume that the photobleaching has little effect on the fluorescence of $[A]$ because the bleached $A$ subunits readily leave the bleached region by diffusion and are replaced by unbleached subunits at an equivalent concentration. Also, it is assumed that the amount of $A$ that is bleached is small compared with the total amount in the cell. Assuming that Eqn A1 has reached equilibrium prior to the bleaching event at $\mathrm{t}=0$, and that $[A]$ and $[B]$ are unaffected by the bleaching process, then

$$
[A]=[A]_{e} \text { and }[B]=[B]_{e}
$$

so that by combining Eqns A4 and A6

$$
K_{\mathrm{on}}=\frac{k_{\mathrm{off}}[A B]_{e}}{[A][B]} .
$$

Substituting Eqn A7 into Eqn A5,

$$
\frac{d[A B]}{d t}=k_{\mathrm{off}}[A][B]_{e}-k_{\mathrm{off}}[A B] .
$$

Assuming that $[\mathrm{AB}]=0$ at $\mathrm{t}=0$, then the solution to equation $\mathrm{A} 8$ is given by

$$
\frac{[A B]}{[A B]_{e}}=1-e^{-k_{\text {off }} t}
$$

which applies regardless of the degree of binding site saturation at equilibrium. Therefore, given the stated assumptions, the rate constant for FRAP recovery is identical to the dissociation rate constant, $k_{\text {off. }}$

The authors are grateful to Dorota Gruber for assistance with the experiments, to Nikki I. Smith and Achyuta Adhvaryu for assistance with data analysis, to Paul Maddox for laser adjustments vital for FRAP, and to individuals at Universal Imaging for providing algorithms used for collecting and analyzing data. This work was supported by grants from NIH to J.C.B. (CA 70951), to C.M.W.-S. (GM61804) and to E.D.S. (GM60678). J.C.B. was an Avon Products Foundation Scholar during a portion of this work. C.M.W.-S. was supported by a Jane Coffin Childs Fellowship and funding from the Institute for Childhood and Neglected Diseases at the Scripps Research Foundation, and D.J.O. was supported by a Biomedical Engineering Research Grant from the Whitaker Foundation.

\section{REFERENCES}

Andersen, S. S. L. (2000). Spindle assembly and the art of regulating microtubule dynamics by MAPs and Stathmin/Op18. Trends Cell Biol. 10, 261-267.

Bershadsky A. D. and Gelfand, V. I. (1981). ATP-dependent regulation of cytoplasmic microtubule disassembly. Proc. Natl. Acad. Sci. USA 78, 36103613.

Blagosklonny, M. V., Chuman, Y., Bergan, R. C. and Fojo, T. (1999). Mitogen-activated protein kinase pathway is dispensable for microtubuleactive drug-induced Raf-1/Bcl-2 phosphorylation and apoptosis in leukemia cells. Leukemia 13, 1028-1036.

Bulinski, J. C. and Borisy, G. G. (1979). Self-assembly of Hela tubulin and the identification of HeLa microtubule-associated proteins. Proc. Natl. Acad. Sci. USA 76, 293-297.

Bulinski, J. C. and Borisy, G. G. (1980). Microtubule-associated proteins from cultured HeLa cells: Analysis of molecular properties and effects on microtubule polymerization. J. Biol. Chem. 255, 11570-11576.

Bulinski, J. C. and Bossler, A. (1994). Purification and characterization of ensconsin, a novel microtubule stabilizing protein. J. Cell Science 107, 2839-2849. 
Bulinski, J. C., McGraw, T., Gruber, D., Nguyen, H. L. and Sheetz, M. P. (1997). Overexpression of MAP4 inhibits organelle motility in vivo. J. Cell Sci. 110, 3055-3064.

Bulinski, J. C., Gruber, D., Faire, K., Prasad, P. and Chang, W. (1999). GFP chimeras of E-MAP-115 (ensconsin) domains mimic behavior of the endogenous protein in vitro and in vivo. Cell Struct. Funct. 24, 313320.

Butner, K. A. and Kirschner, M. W. (1991). Tau protein binds to microtubules through a flexible array of distributed weak sites. J. Cell Biol. 115, 717-730.

Cassimeris, L. (1999). Accessory protein regulation of microtubule dynamics throughout the cell cycle. Curr. Opin. Cell Biol. 11, 134-141.

Chapin, S. and Bulinski, J. C. (1994). Cellular microtubules heterogeneous in their content of MAP4 (210Kd MAP). Cell Motil. Cytoskel. 27, 133-149.

Coffey, R. L. and Purich, D. L. (1995). Non-cooperative binding of the MAP2 microtubule-binding region to microtubules. J. Biol. Chem. 270, 10351040 .

Desagher, S., Osen-Sand, A., Nichols, A., Eskes, R., Montessuit, S., Lauper, S., Maundrell, K., Antonsson, B. and Martinou, J. C. (1999). Bidinduced conformational change of Bax is responsible for mitochondrial cytochrome c release during apoptosis. J. Cell Biol. 144, 891-901.

Ebneth, A., Godemann, R., Stamer, K., Illenberger, S., Trinczek, B. and Mandelkow, E. (1998). Overexpression of tau protein inhibits kinesindependent trafficking of vesicles, mitochondria and endoplasmic reticulum: implications for Alzheimer's disease. J. Cell Biol. 143, 777-794.

Fabré-Jonca, N., Allaman, J.-M., Radlgruber, G., Meda, P., Kiss, J. Z., French, L. E. and Masson, D. (1998). The distribution of murine 115-kDa epithelial MT-associated protein (E-MAP-115) during embryogenesis and in adult organs suggests a role in epithelial polarization and differentiation. Differentiation 63, 169-180.

Fabré-Jonca, N., Viard, I., French, L. E. and Masson, D. (1999). Upregulation and redistribution of E-MAP-115 (epithelial microtubuleassociated protein of $115-\mathrm{kDa}$ ) in terminally differentiating kerarinocytes is coincident with the formation of intercellular contacts. J. Invest. Dermatol. 112, 216-225.

Faire, K., Waterman-Storer, C., Gruber, D., Masson, D., Salmon, E. and Bulinski, J. C. (1999). E-MAP-115 (Ensconsin) associates dynamically with microtubules in vivo and is not a physiological modulator of microtubule dynamics. J. Cell Sci. 112, 4243-4255.

Gruber, D., Faire, K. and Bulinski, J. C. (2001). Abundant expression of the microtubule-associated protein, ensconsin (E-MAP-115), alters the cellular response to Taxol. Cell Motil. Cytoskel. (in press).

Gundersen, G. G., Khawaja, S. and Bulinski, J. C. (1987). Postpolymerization detyrosination of alpha tubulin: a mechanism for subcellular differentiation of microtubules. J. Cell Biol. 105, 251-264.

Hamaguchi, Y., Toriyama, M., Sakai, H. and Hiramoto, Y. (1985). Distribution of fluorescently labeled tubulin injected into sand dollar eggs from fertilization through cleavage. J. Cell Biol. 100, 1262-1272.

Hamill, D. R., Howell, B., Cassimeris, L. and Suprenant, K. A. (1998). Purification of a WD repeat protein, EMAP, that promotes microtubule dynamics through an inhibition of rescue. J. Biol. Chem. 273, 9285-9291.

Jordan, M. A., Thrower, D. and Wilson, L. (1992). Effects of vinblastine, podophyllotoxin and nocodazole on mitotic spindles. Implications for the role of microtubule dynamics in mitosis. J. Cell Sci. 102, 401-416.

Larsson, N., Segerman, B., Howell, B., Fridell, K., Cassimeris, L. and Gullberg, M. (1999). Op18/stathmin mediates multiple region-specific tubulin and microtubule-regulating activities. J. Cell Biol. 146, 1289-1302.

Lepley, D. M. Palange, J. M. and Suprenant, K. A. (1999). Sequence and expression patterns of a human EMAP-related protein-2 (HuEMAP-2). Gene 237, 343-349.

Liao, G., Nagasaki, T. and Gundersen, G. G. (1995). Low concentrations of nocodazole and taxol interfere with fibroblast locomotion without significantly affecting microtubule level: implications for the role of dynamic microtubules in cell locomotion. J. Cell Sci. 108, 3473-3483.

Ling, Y. H., Tornos, C. and Perez-Soler, R. (1998). Phosphorylation of Bcl2 is a marker of $\mathbf{M}$ phase events and not a determinant of apoptosis. J. Biol. Chem. 273, 18984-18991.

Maddox, P. S., Bloom, K. S. and Salmon, E. D. (2000). The polarity and dynamics of microtubule assembly in the budding yeast Saccharomyces cerevisiae. Nat. Cell Biol. 2, 36-41.

Mandelkow, E. and Mandelkow, E. M. (1995). Microtubules and microtubule-associated proteins. Curr. Opin. Cell Biol. 7, 72-81.
Masson, D. and Kreis, T. E. (1993). Identification and molecular characterization of E-MAP-115, a novel microtubule-associated protein predominantly expressed in epithelial cells. J. Cell Biol. 123, 357-371.

McNally, F. J. (1999). Microtubule dynamics: controlling split ends. Curr. Biol. 9, R274-R276.

Mercken, M., Fischer, I., Kosik, K. S. and Nixon, R. A. (1995). Three distinct axonal transport rates for tau, tubulin and other microtubuleassociated proteins: evidence for dynamic interactions of tau with microtubules in vivo. J. Neurosci. 15, 8259-8267.

Nguyen, H. L., Gruber, D. and Bulinski, J. C. (1999). Microtubuleassociated protein 4 (MAP4) regulates assembly, protomer-polymer partitioning and synthesis of tubulin in cultured cells. J. Cell Sci. 112, 18131824.

Olmsted, J. B., Stemple, D. L., Saxton, W. M., Neighbors, B. W. and McIntosh, J. R. (1989). Cell cycle-dependent changes in the dynamics of MAP2 and MAP4 in cultured cells. J. Cell Biol. 109, 211-223.

Olsen, K. R., McIntosh, J. R. and Olmsted, J. B. (1995). Analysis of MAP4 function in living cells using green fluorescent protein (GFP) chimeras. $J$. Cell. Biol. 130, 639-650.

Parysek, L. M., Asnes, C. F. and Olmsted, J. B. (1984). MAP4: occurrence in mouse tissues. J. Cell Biol. 99, 1309-1315.

Perez, F., Diamantopoulos, G. S., Stalder, R. and Kreis, T. E. (1999). CLIP170 highlights growing microtubule ends in vivo. Cell 96, 517-527.

Sauve, D. M., Anderson, H. J., Ray, J. M., James, W. M. and Roberg, M. (1999). Phosphorylation-induced rearrangement of the histone H3 NH2terminal domain during mitotic chromosome condensation. J. Cell Biol. 145, 225-235.

Saxton, W. M., Stemple, D. L., Leslie, R. J., Salmon, E. D., Zavortink, M. and McIntosh, R. J. (1984). Tubulin dynamics in cultured mammalian cells. J. Cell Biol. 99, 2175-2186.

Scherson, T., Kreis, T. E., Schlessinger, J., Littauer, U., Borisy, G. G. and Geiger, B. (1984). Dynamic interactions of fluorescently labeled microtubule-associated proteins in living cells. J. Cell. Biol. 99, 425-434.

Shtil, A. A., Mandlekar, S., Yu, R., Walter, R. J., Hagen, K., Tan, T. H., Roninson, I. B. and Kong, A. N. (1999). Differential regulation of mitogenactivated protein kinases by microtubule-binding agents in human breast cancer cells. Oncogene 8, 377-384.

Stone, A. A. and Chambers, T. C. (2000). Microtubule inhibitors elicit differential effects on MAP kinase (JNK, ERK and p38) signaling pathways in human KB-3 carcinoma cells. Exp. Cell Res. 254, 110-119.

Sun, D., Leung, C. L. and Liem, R. K. H. (2001). Characterization of the microtubule binding domain of Microtubule Actin Crosslinking Factor (MACF): identification of a novel group of microtubule associated proteins. J. Cell. Sci. 114, 161-172.

Suprenant, K. A., Dean, K., McKee, J. and Hake, S. (1993). EMAP, an echinoderm microtubule-associated protein found in microtubule-ribosome complexes. J. Cell Sci. 104, 445-450.

Wadsworth, P. and Salmon, E. D. (1988). Spindle microtubule dynamics: modulation by metabolic inhibitors. Cell Motil. Cytoskel. 11, 97-105.

Wallis, K. T., Azhar, S., Rho, M. B., Lewis, S. A., Cowan, N. J. and Murphy, D. B. (1993). The mechanism of equilibrium binding of microtubule-associated protein 2 to microtubules. Binding is a multi-phasic process and exhibits positive cooperativity. J. Biol. Chem. 268, 1515815167.

Waterman-Storer, C. M. and Salmon, E. D. (1997). Actomyosin-based retrograde flow of microtubules in the lamella of migrating epithelial cells influences microtubule dynamic instability and turnover and is associated with microtubule breakage and treadmilling. J. Cell Biol. 139, 417-434.

Waterman-Storer, C. M. and Salmon, E. D. (1998). How microtubules get fluorescent speckles. Biophys. J. 75, 2059-2069.

Waterman-Storer, C. M., Desai, A., Bulinski, J. C. and Salmon, E. D. (1998). Fluorescent speckle imaging: visualizing the movement, assembly and turnover of macromolecular assemblies in living cells. Curr. Biol. 8, 227-230.

Wolfson, M., Yang, C. P. and Horwitz, S. B. (1997). Taxol induces tyrosine phosphorylation of Shc and its association with Grb2 in murine RAW 264.7 cells. Int. J. Cancer 70, 248-252.

Yujiri, T., Fanger, G. R., Garrington, T. P., Schlesinger, T. K., Gibson, S. and Johnson, G. L. (1999). MEK kinase 1 (MEKK1) transduces c-Jun NH2-terminal kinase activation in response to changes in the microtubule cytoskeleton. J. Biol. Chem. 274, 12605-12610. 